\title{
COMMENT
}

\section{After the gold rush}

Neil Hall*

Have you noticed anything different recently? Last year, something we have all taken for granted for decades changed. I am not talking about new theories on the functionality of 'junk DNA' (I think that has received enough attention already) but the consequences of the change I am referring to may radically affect the way we do science.

A few months ago, the National Human Genome Research Institute (NHGRI) updated their analysis of the cost of sequencing and, for the first time since records began, it got more expensive (Figure 1). You know the graph, the one which looks like the profile of an aquapark waterslide, a gradual incline followed by a precipitous drop as next generation sequencing kicks in. Well, now the waterslide ends with a treacherous upward flick! We have become so comfortable in the knowledge that DNA sequencing reduces in cost at a rate that makes each run cheaper than the last, that some of the scientific community are in denial. I have even seen people present this graph at meetings and explain how sequencing is getting cheaper every day despite the fact they are standing in front of a 10 foot PowerPoint slide showing clearly that this is not true. In fact, the cost of sequencing a human genome increased by $\$ 717$ (an increase of 12\%) between April 2012 and October 2012. This month the new figures showed that the price fell again, but the point remains - you can forget Moore's law!

Some of you will think this merely means you need to replace the opening slide in your PowerPoint deck and tone down some of the rhetoric around $\$ 10$ human genomes and the advent of free sequencing. I, however, think that the long-term ramifications may be more profound.

\section{What follows is deliberately provocative...}

Imagine if microscopes got ten times more powerful every year. You could envisage performing the same experiment every few months and making fresh

\footnotetext{
*Correspondence: neil.hall@liv.ac.uk

The Centre for Genomic Research, The University of Liverpool, Liverpool, L69 7ZB, UK
}

discoveries while staring at the same slide... You would not need new ideas to keep publishing interesting findings. Does this sound at all familiar? It should do, because it's pretty close to what we have been doing in the genomics field for the last 10 years or so.

We, that is genomicists, have been spoiled. We have been real-estate agents working in a housing boom; bankers trading in debt. We have not been made to work; worse still, there has been very little incentive to think. While we have been sliding down the NHGRI sequencingcost graph we have constantly been fed a high-calorie diet of technical improvements that have led to startlingly obvious (but interesting) discovery experiments to perform; experiments that were impossible or too costly only months previously. What do you do after you have sequenced a human genome? Sequence 1,000 human genomes [1]! When you have done that, sequence 2,000 human genomes [2], sequence their microbiomes [3], sequence their transcriptomes [4], sequence Earth [5]. These are all sensible things to do, the only reason they had not been carried out before is because they couldn't. Many (but not all) genomic experiments are not ingenious or elegant, they are brute force discovery projects made possible by clever technology. The technology has been doing the thinking for us. But, as with all exponential trends in ecosystems or economies, the party always has to come to an end.

So what does it mean for us now that the cost-graph has turned against us? Well, for a start, it means that what is possible next year will be the same as what had been possible last year. No new doors will be opened by cheaper, faster sequencing. This means that new ideas will have a higher premium than before. The funders of genomic science will also have to learn to expect more expensive experiments. They have been used to being offered more for less; but now, more will simply cost more. Also, we will have to stop promising a future of cheap genomes that will make our research relevant to the clinic. I have heard many conference presentations where the justification for a specific genomic assay is 'because soon it will be so cheap and easy that every doctor will have one in his office.' Clearly this is not inevitable - certainly not soon. So translation will have to come from what we have now, not what we expect to have tomorrow. 


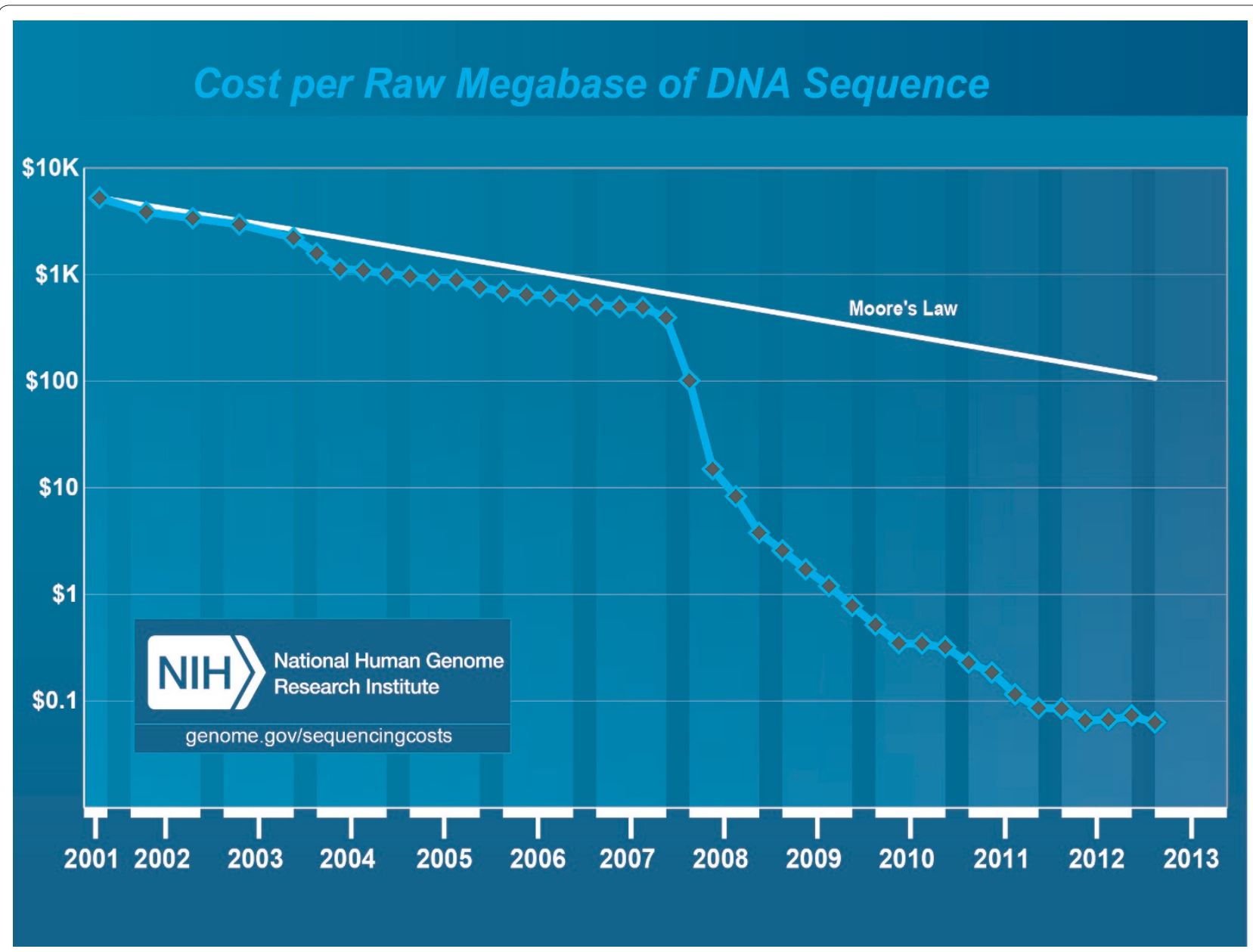

Figure 1. Changes in the cost of sequencing over time. The change in sequencing costs over time at sequencing centers funded by the National Human Genome Research Institute (NHGRI), up until January 2013. The cost displayed is per raw megabase of sequence. Data are provided by the NHGRI Genome Sequencing Program, with full details available at [7]. @ NHGRI; reproduced with permission.

\section{Chasing the illusive hangover cure}

'But...' I hear you scream, '...this is a temporary blip. Soon we will be saved by new cool technology that will plug into my laptop and sequence a genome for $\$ 10$ in an hour.' In reality, is this just something we simply want to believe? There really is no reason to think that sequencing methodology is about to undergo a revolution in the near future.

I am always amazed at the self-inflicted hype that follows any hint of a story where some company has come across a new way of sequencing that is going to turn all our Illumina kits into oversized doorstops. Often this comes not from the companies themselves but the scientists who are so desperate to buy them. The hype is usually followed by hyper-critical twitter and blog commentaries when the machine in question does not appear to do what we want it to (see this revealing interview with Oxford Nanopore's Clive Brown [6]), in a cycle that has repeated itself at least three times in the last 5 years. I begin to wonder why we don't learn from history.

Like addicts chasing a high, our anger at being denied a technology fix is born out of irrational desperation. The fact of the matter is that even if Oxford Nanopore (or someone) were to deliver a revolutionary technology this year, the crunch will happen soon. At some point, the exponential decrease in sequencing costs will stop again and we will have to work with a static technology base for a prolonged period.

\section{Embracing the pain}

Sometimes what is good for you hurts; like exercise, vaccinations and teaching. Any decent economist will tell you - stability is generally a good thing, and genomics could benefit from a time of reflection and consolidation, where we can simply plan for the long-term without the threat of disruptive technologies instantly obsoleting our efforts. The losers in this stagnation of costs may well be 
the 'big science' experiments. I have no doubt that they tend to seem cooler to funders in a scenario where the sample numbers are forever increasing owing to decreasing data generation costs. However, I for one am more excited by a world where, instead of chasing bigger numbers and deeper coverage, we invest more resources into exploring the true potential of the technology that we currently have. And if we stop looking for a new technology on the horizon, maybe one will turn up.

\section{Acknowledgements}

The change in the direction of sequencing costs was brought to my attention by a tweet. Unfortunately, I can't remember who from but it was either @ BioMickWatson or @lexnederbragt.

Published: 7 May 2013

References

1. The 1000 Genome Project Consortium: An integrated map of genetic variation from 1,092 human genomes. Nature 2012, 491:56-65.
2. GenomeWeb: 1000 Genomes Project Releases Pilot Project Data, Plans to Sequence 2,500 Genomes in Total [http://www.genomeweb.com/ sequencing/1000-genomes-project-releases-pilot-project-data-planssequence-2500-genomes-tot]

3. The Human Microbiome Project Consortium: Structure, function and diversity of the healthy human microbiome. Nature 2012, 486:207-214

4. Djebali S, Davis CA, Merkel A, Dobin A, Lassmann T, Mortazavi A, Tanzer A, Lagarde J, Lin W, Schlesinger F, Xue C, Marinov GK, Khatun J, Williams BA, Zaleski C, Rozowsky J, Röder M, Kokocinski F, Abdelhamid RF, Alioto T, Antoshechkin I, Baer MT, Bar NS, Batut P, Bell K, Bell I, Chakrabortty S, Chen X Chrast J, Curado J, et al.: Landscape of transcription in human cells. Nature 2012, 489:101-108.

5. The Earth Microbiome Project [http://www.earthmicrobiome.org/]

6. Loman N: A chat with Oxford Nanopore's Clive Brown at AGBT 2013 [http://pathogenomics.bham.ac.uk/ blog/2013/03/a-chat-with-oxford-nanopores-clive-brown-at-agbt-2013/]

7. National Human Genome Research Institute: DNA Sequencing Costs [http://www.genome.gov/sequencingcosts/]

doi:10.1186/gb-2013-14-5-115

Cite this article as: Hall N: After the gold rush. Genome Biology 2013, 14:115. 